\title{
The Therapeutic Effects of Yoga on Health and Related Diseases: An Analytical Review
}

\author{
Gurmeet Singh Sarla ${ }^{1}$ \\ ${ }^{1}$ Senior Registrar, 159 General Hospital, India
}

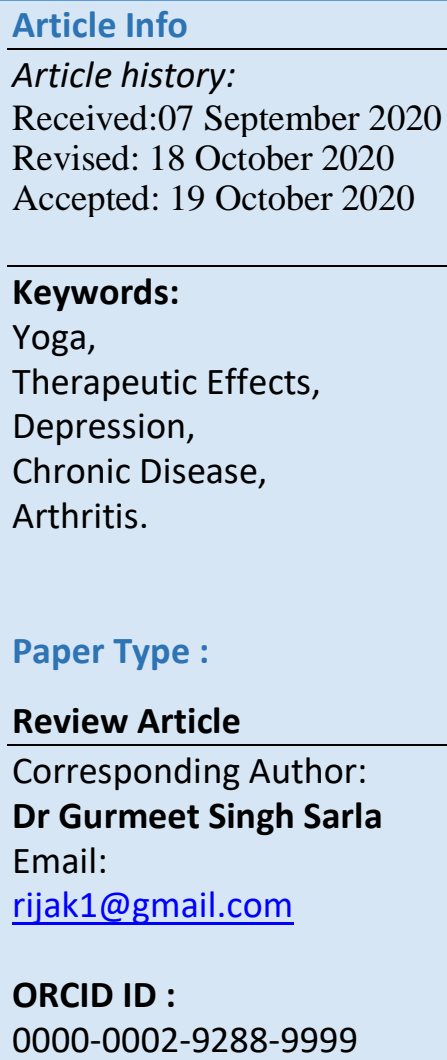

\begin{abstract}
Purpose: Yoga means unity of mind and body. It has an effective role in reducing stress, anxiety, and depression and thus it can be used as complementary medicine. It not only is effective for strengthening the body but also incorporates appropriate breathing techniques, mindfulness, and meditation in order to achieve the maximum benefits. The objective of this study is to assess the findings of selected articles regarding the therapeutic effects of yoga and to provide a comprehensive review of the benefits of regular yoga practice.

Subjects and Methods: In order to locate research studies and interventions that examined the therapeutic effects of yoga, databases were searched through Google Scholar and PubMed. The keywords entered into the database were Yoga, therapeutic effects, stress, anxiety, depression, chronic disease, arthritis, low backache, diabetes, cancer and pregnancy and articles from 1993 to 2007 were searched.

Results: It is revealed that Yoga improves physical, mental, intellectual and spiritual health and offers an effective method of managing and reducing stress, anxiety and depression. It also triggers neurohormonal mechanisms that bring about health benefits, evidenced by the suppression of sympathetic activity. Yoga should be considered as a complementary therapy or alternative method for medical therapy in the treatment of stress, anxiety, depression, and other mood disorders as it has been shown to create a greater sense of well-being, increase feelings of relaxation, improve self-confidence and body image, improve efficiency, better interpersonal relationships, increase attentiveness, lower irritability, and encourage an optimistic outlook on life.

Conclusion: Yoga teaches us to slow down, quieten our minds and connect with our inner selves which ultimately help in relieving us from the pressures and stressors of this modern day life.
\end{abstract}

\section{Introduction}

Yoga is a Sanskrit word meaning solidarity of mind and body. Studies have exhibited impact of yoga on numerous conditions, including multiple sclerosis, asthma, irritable bowel syndrome, lymphoma, hypertension, drug addiction, osteoarthritis, and mental health issues. Yoga has a beneficial role in reducing stress, anxiety, and depression and thus it can be used as complementary medicine. Complementary medicine refers to a category of treatments and interventions that have not been raised in modern medicine (Borji et al., 2017).

Yoga not only is successful in strengthening the body but also incorporates appropriate breathing techniques, mindfulness, and meditation in order to accomplish health benefits. The physical activities (asanas) may increase the patient's physical adaptability, coordination, and strength, while the breathing practices and meditation may quieten and 
focus the brain to create more noteworthy mindfulness and reduce anxiety(Kirkwood et al., 2005). Other valuable impacts may include a reduction of distress, blood pressure, and improvements in resilience, mood, and metabolic regulation (Yang, 2007). Yoga gives an elective way to deal with health that is characteristic, low-tech, moderately modest and for the most part very safe ( Khalsa et al., 2016).

Objective: The goal of this review article is to survey the findings of chosen articles with respect to the helpful impacts of yoga and to give an extensive audit of the advantages of customary yoga practice.

\section{Methodology and Procedures}

In order to look into studies and mediations that inspected the restorative impacts of yoga, databases were looked through PubMed and Google Scholar. The catchphrases went into the database were Yoga, therapeutic effects, stress, anxiety, depression, chronic disease, arthritis, low backache, diabetes, cancer and pregnancy and more than 25 articles from 1993 to 2007 were searched. All articles on yoga which mentioned therapeutic and health effects were included and articles which deliberated upon the asanas and the way to perform them were excluded.

\section{Results and Discussion}

The word "yoga" comes from a Sanskrit root "yuj" which means union, or to join and to direct and concentrate one's attention (Lasater, 1997). Regular practice of yoga advances quality, continuance, adaptability and encourages attributes of benevolence, empathy, and more prominent discretion, while developing a feeling of smoothness and well-being (Collins, 1998). The act of yoga delivers a physiological state inverse to that of the flight-orfight pressure reaction and with that break in the pressure reaction, a feeling of parity and relationship between the mind and body can be achieved (Arora, 2008).Yoga is perceived as a type of mind-body prescription that incorporates a person's physical, mental and otherworldly segments to improve parts of wellbeing, especially stress related illnesses (Atkinson \& Permuth- Levine, 2009).

\section{Health Effects of Yoga}

Yoga-based relaxation training normalizes the function of the autonomic nervous system by deviating both sympathetic and parasympathetic indices toward more "normal" middle region of the reference values (Pilkington et al., 2005). Yoga fundamentally diminishes pulse and systolic and diastolic blood pressures (McCaffrey et al., 2005). Studies show that yoga diminishes blood glucose levels (Khatri et al., 2007) .Yoga has been appeared to have prompt mental impacts: decreasing anxiety and increasing feelings of emotional, social, and spiritual well-being (Gupta et al., 2006; Moadel et al., 2007).

\section{How does Yoga work?}

Yoga brings the autonomic nervous system into healthy balance by invigorating the 
parasympathetic system. The sympathetic nervous system or our "emergency response system," is activated when our body or mind feels threatened or perceives being stressed. This "flight or fight" response results in vasoconstriction, causing decreased blood flow to the extremities and the digestive system in order to prepare one for survival. Conversely, the parasympathetic framework is animated when one unwinds; it is regularly called the "rest and digest" component of our sensory system. The parasympathetic system stimulates blood flow to the digestive system, brain, extremities and sexual organs. Yoga lessens physiologic excitement and quietens down this consistent play of the autonomic framework decreasing pulse and circulatory strain and facilitates breath-all indications of improved parasympathetic tone (Stearns et al., 2010).

Yoga-based practices of postures (asanas) and movement sequences are usually taught in conjunction with some type of breathing and/or meditation technique (Khalsa et al., 2016). This type of mindful movement with slow, rhythmic breathing is more likely to promote parasympathetic and vagal tone compared to other forms of exercise. Yogic breathing procedures - specifically, exchange nostril breathing, which includes breathing through the left and right nostril-has been related with expanded parasympathetic movement and diminished systolic blood pressure. Slow and cadenced breathing has likewise been appeared to advance the release of prolactin and oxytocin, which can cultivate sentiments of companionship, serenity and bonding to others which are released during childbirth which help the mother relax and bond with her new-born during the very painful process of child birth (Khalsa et al., 2016).

\section{Effects on Neurotransmitters}

Yoga increases neurotransmitters including GABA, serotonin, and dopamine which are natural anti-depressants. Yoga also increases the levels of melatonin, helping to initiate sleep, improving sleep quality and sleep regulation, as well as increasing levels of oxytocin, the "bonding hormone", thus helping with feelings of connectedness and "being seen and heard" (Lipschitz et al., 2015 ).

\section{Effects on inflammation}

Yoga has been found to decrease inflammatory markers such as C-reactive protein and other inflammatory cytokines in the blood, while increasing levels of multiple immunoglobulins and natural killer cells.

\section{Yoga in Low -Backache and Arthritis}

Yoga incorporates important elements of body awareness such as proprioception, coordination, balance and postural alignment, all of which are particularly important in individuals with joint disease (Exercise and Arthritis, 2017).Yoga as an intervention for chronic low backache has been found to be beneficial in short-term improvements in functional disability(Holtzman, 2013), and has improved pain, back function, spinal mobility, depression and anxiety in patients with chronic low backache(Tekur,2012). 


\section{Effects on Heart}

According to the American Heart Association, yoga practices can help to lower blood pressure, increase lung capacity, improve respiratory function and heart rate, improve circulation and boost muscle tone (Yoga and Heart Health, 2017). Studies have uncovered that the general impact of yoga brings about a decrease of systolic BP of around $10 \mathrm{mmHg}$ and roughly a $8 \mathrm{mmHg}$ decrease in diastolic BP (Cramer, 2016).

\section{Effects on body weight and lipid profile}

Yoga has been found to be particularly helpful in the management of obesity. A study has revealed that practicing yoga for a year helped significant improvements in the ideal body weight (Bera \& Rajapurkar, 1993). The regular practice of yoga has shown to improve the serum lipid profile in the patients with known ischemic heart disease as well as in healthy subjects (Mahajan et al., 1999).

\section{Effects on Diabetes Mellitus}

A study has shown that there occurs a decrease in the need for oral hypoglycemic agents to maintain adequate blood sugar control in the population that practiced yoga(Jain et al., 1993 ).Another study observed that the performance of Yoga asanas led to increased sensitivity of B cells of the pancreas to the glucose signal(Manjunatha et al., 2005 ).

\section{Effects on pregnancy}

A study observed that yoga practices including physical postures, breathing, and meditation practiced by pregnant women $1 \mathrm{~h}$ daily resulted in an increase in birth weight, decrease in preterm labour, and decrease in IUGR(Narendran et al., 2005 ).

\section{Effects on Mood}

Yoga has demonstrated improvements in mood and quality of life for elderly people and improvements in perceived stress, depression, anxiety, energy, fatigue, and overall wellbeing of the individual. In addition, it was proved to be effective in relieving emotionallydistressed women (Brown et al., 2005).

\section{Cancer}

Yoga is beneficial for people with cancer in managing symptoms such as fatigue, insomnia, mood disturbances and stress, and improving quality of life (Distasio, 2008).

\section{Conclusion and Suggestion}

Yoga improves physical, mental, intellectual and spiritual well being and offers a powerful strategy to diminish stress, anxiety and depression. It likewise triggers neurohormonal components that achieve medical advantages, confirmed by the concealment of sympathetic activity. Yoga ought to be considered as a complementary therapy or alternative method for medical therapy in the treatment of stress, anxiety, depression, and 
other mood disorders as it has been shown to create a greater sense of well-being, increase feelings of relaxation, improve self-confidence and body image, improve efficiency, better interpersonal relationships, increase attentiveness, lower irritability, and encourage an optimistic outlook on life. Yoga instructs us to back off, quieten our brains and associate with our internal identities which eventually encourage us to assuage us from the weights and stressors of this advanced distressing life.

\section{Conflict of Interest}

The author of the article declares no conflict of interest.

\section{Funding:}

The author received no funding for conducting this study.

\section{References}

Arora, S., \& Bhattacharjee, J. (2008). Modulation of immune responses in stress by Yoga. International journal of yoga, 1(2), 45.

Atkinson, N. L., \& Permuth-Levine, R. (2009). Benefits, barriers, and cues to action of yoga practice: A focus group approach. American journal of health behavior, 33(1), 3-14.

Bera, T. K., \& Rajapurkar, M. V. (1993). Body composition, cardiovascular endurance and anaerobic power of yogic practitioner. Indian Journal of Physiology and Pharmacology, 37, 225-225.

Bir, S. (Ed.). (2016). Principles and Practice of Yoga in Health Care. Handspring Publishing Limited.

Borji, M., Otaghi, M., Salimi, E., \& Sanei, P. (2017). Investigating the effect of performing the quiet time protocol on the sleep quality of cardiac patients.

Cramer, H. (2016). The efficacy and safety of yoga in managing hypertension. Experimental and Clinical Endocrinology \& Diabetes, 124(02), 65-70.

Collins, C. (1998). Yoga: Intuition, preventive medicine, and treatment. Journal of Obstetric, Gynecologic, \& Neonatal Nursing, 27(5), 563-568.

DiStasio, S. A. (2008). Integrating yoga into cancer care. Clinical Journal of Oncology Nursing, 12(1).

Gupta, N., Khera, S., Vempati, R. P., Sharma, R., \& Bijlani, R. L. (2006). Effect of yoga based lifestyle intervention on state and trait anxiety. Indian journal of physiology and pharmacology, 50(1), 41.

Exercise and Arthritis. [(accessed on 30 January 2017)]. Available

online: http://www.rheumatology.org/I-Am-A/Patient-Caregiver/Diseases-

Conditions/Living-Well-with-Rheumatic-Disease/Exercise-andArthritis\#sthash.2dxYmjdw.dpufRA.

Jain, S. C., Uppal, A., Bhatnagar, S. O. D., \& Talukdar, B. (1993). A study of response pattern of non-insulin dependent diabetics to yoga therapy. Diabetes Research and clinical practice, 19(1), 69-74. 
Holtzman, S., \& Beggs, R. T. (2013). Yoga for chronic low back pain: a meta-analysis of randomized controlled trials. Pain Research and Management, 18.

Kriya, B. R. G. P. S. yogic breathing in the treatment of stress, anxiety and depression. Part I: neurophysiologic model. J. Altern. Complement. Med. 2005a, 11, 189-201.

Kirkwood, G., Rampes, H., Tuffrey, V., Richardson, J., \& Pilkington, K. (2005). Yoga for anxiety: a systematic review of the research evidence. British journal of sports medicine, 39(12), 884-891.

Khatri, D., Mathur, K. C., Gahlot, S., Jain, S., \& Agrawal, R. P. (2007). Effects of yoga and meditation on clinical and biochemical parameters of metabolic syndrome. Diabetes Research and Clinical Practice, 78(3), e9-e10.

Lasater J.(1997) The heart of pantajali. Yoga J. 137:134-44.

Lipschitz, D. L., Kuhn, R., Kinney, A. Y., Grewen, K., Donaldson, G. W., \& Nakamura, Y. (2015). An exploratory study of the effects of mind-body interventions targeting sleep on salivary oxytocin levels in cancer survivors. Integrative cancer therapies, 14(4), 366-380.

McCaffrey, R., Ruknui, P., Hatthakit, U., \& Kasetsomboon, P. (2005). The effects of yoga on hypertensive persons in Thailand. Holistic nursing practice, 19(4), 173-180.

Moadel, A. B., Shah, C., Wylie-Rosett, J., Harris, M. S., Patel, S. R., Hall, C. B., \& Sparano, J. A. (2007). Randomized controlled trial of yoga among a multiethnic sample of breast cancer patients: effects on quality of life. Journal of Clinical Oncology, 25(28), 4387-4395.

Mahajan, A. S., Reddy, K. S., \& Sachdeva, U. (1999). Lipid profile of coronary risk subjects following yogic lifestyle intervention. Indian heart journal, 51(1), 37-40.

Manjunatha, S., Vempati, R. P., Ghosh, D., \& Bijlani, R. L. (2005). An investigation into the acute and long-term effects of selected yogic postures on fasting and postprandial glycemia and insulinemia in healthy young subjects. Indian journal of physiology and pharmacology, 49(3), 319.

Narendran, S., Nagarathna, R., Narendran, V., Gunasheela, S., \& Nagendra, H. R. R. (2005). Efficacy of yoga on pregnancy outcome. Journal of Alternative \& Complementary Medicine, 11(2), 237-244.

NurrieStearns, M., \& NurrieStearns, R. (2010). Yoga for anxiety: Meditations and practices for calming the body and mind. New Harbinger Publications.

Penetrating Postures: The Science of Yoga. (2017). Available online: https://www.forbes.com/sites/alicegwalton/2011/06/16/penetrating-posturesthe-science-of-yoga/?client=safari.

Pilkington, K., Kirkwood, G., Rampes, H., \& Richardson, J. (2005). Yoga for depression: the research evidence. Journal of affective disorders, 89(1-3), 13-24.

Telles, S., Sharma, S. K., \& Balkrishna, A. (2014). Blood pressure and heart rate variability during yoga-based alternate nostril breathing practice and breath awareness. Medical science monitor basic research, 20, 184.

Tekur, P., Nagarathna, R., Chametcha, S., Hankey, A., \& Nagendra, H. R. (2012). A 
comprehensive yoga programs improves pain, anxiety and depression in chronic low back pain patients more than exercise: an RCT. Complementary therapies in medicine, 20(3), 107-118.

Yang, K. (2007). A review of yoga programs for four leading risk factors of chronic diseases. Evidence-Based Complementary and Alternative Medicine, 4.

Yoga and Heart Health. [(accessed on 31 January 2017)]. Available online: http://www.heart.org/HEARTORG/HealthyLiving/PhysicalActivity/Yogaand-Heart-Health_UCM_434966_Article.jsp\#. 